\title{
NOUVELLE MÉTHODE RAPIDE DE DÉTERMINATION DE L'ACIDITÉ DE LA CRÈME, A L'ARRIVÉE \\ A LA centrale VAudoise du Beurre u.L.V.
}

\author{
par \\ R. GOLAY \\ Chef du Laboratoire C.V.B.

L'expérience l'ayant démontré, seule la qualité irréprochable de la crème permet de fabriquer un beurre de premier choix, aromatique et se conservant pendant plusieurs mois. L'examen organoleptique, effectué lors du prélèvement de l'échantillon pour le paiement de celle-ci selon la matière grasse, et la détermination de sa qualité, en laboratoire, ont donc pour but de déceler immédiatement avant la pasteurisation, toute crème présentant un défaut. Compétent, consciencieux, l'employé chargé de ce travail, renseigné par le laboratoire, détermine avec exactitude les crèmes présentant un léger défaut (rinçures, odeur d'étable, goût de fourrage, légèrement acide). Le défaut le plus répandu est certainement la crème légèrement acide. C'est pourquoi, le résultat du laboratoire étant connu trop tard et pour faciliter une appréciation très exacte, nous avons mis à la disposition de cet employé depuis huit années, une méthode pratique rapide, ne demandant pas de travail supplémentaire digne de ce nom, afin d'améliorer ce dépistage.

Plusieurs méthodes de laboratoire permettent de déterminer rapidement l'acidité du lait ou de la crème. Nous citerons pour mémoire :

1.1. Le papier Duplex de Klotz.

1.2. La cuisson.

1.3. L'alcool.

De ces trois méthodes, aucune n'entre en considération.

1.4. La soude caustique ( $\mathrm{NaOH} \mathrm{n} / 4)$ avec phénolphtaléine à 2 p. 100 .

1.5. L'alizarol.

1.6. Détermination $d u \quad p \mathrm{H}$ (avec papier indicateur Lyphan ou appareil $p \mathrm{H}$ avec électrodes).

1.7. Bleu de bromothymol.

1.8. Pourpre de bromocrésol. 


\section{$-2-$}

La soude caustique avec la phénolphtaléine à 2 p. 100 est une méthode exacte, employée en laboratoire. Elle ne pourrait en aucun cas être remise telle quelle à notre praticien. C'est pourquoi, nous avions modifié la méthode de la soude caustique, il y a une quinzaine d'années, en préparant une solution de soude caustique contenant déjà la phénolphtaléine et réglée au degré d'acidité $8 \mathrm{~S}-\mathrm{H}$ pour une certaine quantité de crème. Toutes les crèmes de bonne qualité, ayant une acidité inférieure à $8^{\circ} \mathrm{S}-\mathrm{H}$ se coloraient en rose; toutes les crèmes avec une acidité supérieure à $8^{\circ} \mathrm{S}-\mathrm{H}$ restaient blanches. Malheureusement, la phénolphtaléine étant un réactif se modifiant rapidement, la coloration de la solution disparaissait après quelques jours.

2.1. L'alizarol est une méthode de détermination de l'acidité du lait par virage colorimétrique d'une solution chimique. Elle nous a paru compliquée pour être adaptée au service de la crème.

2.2. Le contrôle du $p H$ de la crème peut se faire avec une bande de papier colorimétrique "Lyphan" ou avee un appareil à électrodes. Nous avons soumis les deux procédés à nos praticiens, mais sans résultat.

2.3. Le bleu de bromothymol a été essayé par notre laboratoire. Par suite de son point de virage situé à $p H \mathbf{H} 7,05$, il n'a pas donné de résultats supérieurs au pourpre de bromo-crésol. Le rouge de chloro-phénol mériterait d'être essayé systématiquement.

\section{3 - Micro-méthode au pourpre de bromo-crésol}

\subsection{Antécédence.}

Il n'est pas de notre propos de faire l'historique de cette substance, réactif chimique, dont voici la formule communiquée obligeamment par la maison Siegfried S.A., fabrique de produits pharmaceutiques à Zofingue (Suisse).

Pourpre de bromocrésol.

5,5' dibromo- o- cresolsulfophtaléine.<smiles>CC1=CC(=C(C2C=C(C)C(O)=C(Br)C2)C2CCCCC2S(=O)(=O)O)C=C(Br)C1=O</smiles>

Il s'agit done d'un dérivé de l'ortho-crésol. 
La "Verbands-Molkerei Bern" nous a fait parvenir au début de 1952, une notice explicative de la méthode mise au point chez eux et consistant :

a) Une solution de pourpre de bromo-crésol $(0,1 \mathrm{~g}$ d'indicateur est dissous dans $250 \mathrm{~cm}^{3}$ d'eau distillée plus $4,1 \mathrm{~cm}^{3} \mathrm{NaOH} \mathrm{n} / 20$ ). Cette solution est indiquée dans « Lüthi Max. Tabellen für Chemiher und Physiker. Basel, 48 ».

b) Une plaque en émail avec rangée de renflements demisphériques, dans lesquels une goutte de la solution était déposée. Avec une anse de platine, un millième de $\mathrm{cm}^{3}$ de crème est déposé sur cette goutte de solution, puis mélangé. Suivant la qualité de la crème, douce ou acide, une coloration se produit.

\subsection{Critique.}

Les quantités sont exactes et la solution prend la couleur pourpre après addition de la soude $\mathrm{n} / 20$.

Le virage de la coloration, selon indication goutte et anse de platine, se fait de la manière suivante :

$6 \circ \mathrm{S}-\mathrm{H}$ : coloration bleue.

$70 \mathrm{~S}-\mathrm{H}$ : coloration bleue.

$8^{\circ} \mathrm{S}-\mathrm{H}$ : coloration bleue.

9० S-H : coloration bleue.

$10^{\circ} \mathrm{S}-\mathrm{H}$ : coloration pourpre.

$12 \circ \mathrm{S}-\mathrm{H}$ : coloration pourpre.

$15^{\circ} \mathrm{S}-\mathrm{H}$ : coloration pourpre.

$20^{\circ} \mathrm{S}-\mathrm{H}$ : coloration jaune.

Virage de la solution avec mélange de $1 \mathrm{~cm}^{3}$ de solution et $1 \mathrm{~cm}^{3}$ de crème :

$6^{\circ} \mathrm{S}-\mathrm{H}$ : coloration bleue.

$70 \mathrm{~S}-\mathrm{H}$ : coloration bleue.

$80 \mathrm{~S}-\mathrm{H}$ : coloration bleue.

$9 \circ \mathrm{S}-\mathrm{H}$ : coloration bleue.

$10^{\circ} \mathrm{S}-\mathrm{H}$ : coloration jaune-gris qui devient bleue après $5 \mathrm{~min}$.

$12 \circ \mathrm{S}-\mathrm{H}$ : coloration jaune-gris.

$20^{\circ} \mathrm{S}-\mathrm{H}$ : coloration jaune.

Le virage de la couleur de la solution est trop tardif avec $10^{\circ} \mathrm{S}-\mathrm{H}$. Il devrait y avoir un virage à $8 \circ \mathrm{S}-\mathrm{H}$.

\subsection{Nouvelle micro-méthode.}

Matériel. Une ou plusieurs étagères en aluminium pour contenir les éprouvettes.

Des éprouvettes graduées à $10 \mathrm{~cm}^{3}$. crème.

Une seringue de 1 ou $5 \mathrm{~cm}^{3}$ permettant de prélever $1 \mathrm{~cm}^{3}$ de

Un litre de solution de pourpre de bromo-crésol. 


\subsection{Manière de procéder.}

Avant le prélèvement des échantillons, chaque tube de l'étagère sera rempli de $10 \mathrm{~cm}^{3}$ de la solution de pourpre de bromo-crésol. Chaque fois que l'employé n'est pas sûr du défaut constaté, il pourra rapidement déterminer l'acidité de la crème; il suffit de prélever $1 \mathrm{~cm}^{3}$ de crème avec la seringue, le vider dans le tube, de brasser et comparer la couleur obtenue. Toutes les crèmes donnant une coloration jaune ont une acidité supérieure à $7,5^{\circ} \mathrm{S}-\mathrm{H}$. Toutes les crèmes faisant apparaître une coloration bleue, ont une acidité de $7,5^{\circ} \mathrm{S}-\mathrm{H}$ et au-dessous.

\subsection{Coloration étalon.}

Au moment du remplissage des tubes, avant le prélèvement des échantillons, on prendra une crème de bonne qualité, d'une acidité contrôlée de $6^{\circ} \mathrm{S}-\mathrm{H}$. On ajoutera $1 \mathrm{~cm}^{3}$ de cette crème dans 3 tubes avec solution. On brassera, puis on ajoutera dans le deuxième $0,1 \mathrm{~cm}^{3}$ d'acide chlorhydrique $\mathrm{n} / 40$ et $0,2 \mathrm{~cm}^{3}$ d'acide chlorhydrique $\mathrm{n} / 40$ dans le troisième. On brassera à nouveau et l'on obtiendra les colorations correspondant à $6^{\circ} \mathrm{S}-\mathrm{H}, 7^{\circ} \mathrm{S}-\mathrm{H}$ et $80 \mathrm{~S}-\mathrm{H}$ permettant d'habituer l'œil aux colorations limites. Les colorations obtenues resteront inchangées pendant $12 \mathrm{~h}$.

\subsection{Préparation de la solution de pourpre de bromo-crésol.}

A 1 litre d'eau distillée (déminéralisée ou bi-distillée) on ajoutera $0,055 \mathrm{~g}$ de pourpre de bromo-crésol en poudre. On laissera la poudre se dissoudre pendant 48 heures, ce laps de temps écoulé, on remplira 10 tubes avec $10 \mathrm{~cm}^{3}$ de la solution pour nous permettre de déterminer exactement le point de virage de la solution, lequel se produit au $p H$ 6,15. Ce $p H$ correspond à l'ancienne méthode; celui de la nouvelle se situe entre $p H$ 5,50-5,80. On titrera avec exactitude l'acidité d'une crème de bonne qualité, laquelle aura si possible, $6 \circ \mathrm{S}-\mathrm{H}$. On ajoutera $1 \mathrm{~cm}^{3}$ de cette crème avec une seringue, dans chaque tube. On brassera. On ajoutera en partant du deuxième tube à partir de la gauche, $0,1 \mathrm{~cm}^{3}$ d'acide chlorhydrique $\mathrm{n} / 40$, puis $0,2 \mathrm{~cm}^{3} ; 0,3 \mathrm{~cm}^{3} ; 0,4 \mathrm{~cm}^{3} ; 0,5 \mathrm{~cm}^{3} ; 0,6 \mathrm{~cm}^{3} ; 0,7 \mathrm{~cm}^{3}$; $0,8 \mathrm{~cm}^{3}$ et $0,9 \mathrm{~cm}^{3}$, correspondant à $6^{\circ} \mathrm{S}-\mathrm{H}, 7 \circ \mathrm{S}-\mathrm{H}, 8 \circ \mathrm{S}-\mathrm{H}, 9 \circ \mathrm{S}-\mathrm{H}$, $10^{\circ} \mathrm{S}-\mathrm{H}, 11^{\circ} \mathrm{S}-\mathrm{H}, 12^{\circ} \mathrm{S}-\mathrm{H}, 13^{\circ} \mathrm{S}-\mathrm{H}, 14^{\circ} \mathrm{S}-\mathrm{H}$ et $15^{\circ} \mathrm{S}-\mathrm{H}$. On brassera et nous obtiendrons une coloration décroissante du bleu au bleu pâle, puis une coloration croissante du jaune pâle au jaune. Le virage entre les deux couleurs doit se situer entre $11^{\circ}-12^{\circ} \mathrm{S}-\mathrm{H}$, ou éventuellement entre $12^{\circ}-13^{\circ} \mathrm{S}-\mathrm{H}$, suivant la qualité de l'eau distillée employée. Pour obtenir le virage de la crème à $7,5^{\circ} \mathrm{S}-\mathrm{H}$, on ajoutera une quantité proportionnelle d'acide chlorhydrique n/10 permettant de combler la différence de titrage. Si, par exemple, le virage s'est produit entre $12^{\circ}-13^{\circ} \mathrm{S}-\mathrm{H}$, on ajoutera la valeur de $5^{\circ} \mathrm{S}-\mathrm{H}$ en acide chlorhydrique $\mathrm{n} / 10$, ce qui permettra d'effec- 
tuer le virage à $12,5^{\circ} \mathrm{S}-\mathrm{H}$ réellement $\left(7,5^{\circ} \mathrm{S}-\mathrm{H}\right.$ acide lactique + $5^{\circ} \mathrm{S}-\mathrm{H}$ acide chlorhydrique) alors qu'il s'effectuera effectivement à $7,5^{\circ} \mathrm{S}-\mathrm{H}$ pour la crème.

La quantité d'acide chlorhydrique $\mathrm{n} / 10$ à ajouter est de $2,5 \mathrm{~cm}^{3}$ par degré et par litre de solution.

Les acides acétique, citrique, lactique, formique, sulfurique, nitrique, etc., peuvent être employés. Les acides forts permettent une meilleure coloration.

\subsection{Critique de la méthode.}

La crème étant un liquide opaque, l'acidité de celle-ci ayant une croissance minime, les différences de coloration apparaissent difficilement. C'est pourquoi le rapport 1-10 a été choisi pour la crème et la solution. Cette méthode permet d'abaisser à $7,5^{\circ} \mathrm{S}-\mathrm{H}$ le virage de la solution et de reconnaître du même coup une crème ayant une acidité semblable. Rien ne s'oppose de titrer à $6,5^{\circ} \mathrm{S}-\mathrm{H}$, il suffirait d'ajouter la valeur d'un degré d'HCl n/10 en plus par litre ; par contre, la coloration étant très pâle, il faudra un employé vraiment bien exercé.

Pendant le temps d'essai, celui-ci a fait apparaître les dérangements suivants :

a) Oxydo-réduction de la poudre de pourpre de bromo-crésol.

La solution sera toujours titrée et contrôlée régulièrement. Après 2 ou 3 ans, celle-ci ne doit plus être employée (la poudre).

b) La solution n'étant pas tamponnée, suivant la qualité de l'eau distillée, celle-ci se dégénère. On pourra compter avec les limites suivantes :

1. Eau distillée. (N'a pas été contrôlée régulièrement.)

2. Eau bi-distillée : minimum 6 mois.

3. Eau déminéralisée qualité $\mathrm{Ph}$. H.V. : minimum 4 mois.

4. Eau déminéralisée qualité ordinaire : 2-3 mois.

\subsection{Conclusion.}

Cette méthode permet de titrer l'acidité de la crème rapidement à son arrivée dans une centrale beurrière, avec un minimum de temps et de travail ; l'exactitude est élevée.

Elle ne remplace pas l'examen organoleptique, celui-ci restant indispensable pour d'autres défauts de la crème.

Seul le souci d'exactitude et d'amélioration de la qualité du beurre a prédominé lors de l'élaboration de cette nouvelle méthode, valable entre 20 et 35 p. 100 de matière grasse.

\section{4. - Résumé}

Nouvelle méthode rapide et pratique de détermination de l'acidité de la crème à l'arrivée dans une centrale beurrière. Cette 
nouvelle méthode, à base d'une solution de pourpre de bromocrésol, permet de déterminer rapidement l'acidité de la crème à l'arrivée dans une centrale beurrière, avec une seringue et des éprouvettes remplies à l'avance.

\section{1. - Summary}

New rapid and practical method of determination creamacidity of arrival in a butter-producingstation. That new method, at base a bromocresolpurplesolution, allow to determine quickly the creamacidity at arrival in a butter-producingstation, with a syringe and test tubes fill up at advance. 